\title{
CT Scan of Pediatric Liver Tumors
}

\author{
Qian Dong ${ }^{1}$ and Jingjing Chen ${ }^{2}$ \\ ${ }^{1}$ Department of pediatric surgery, \\ ${ }^{2}$ Department of radiology The Affiliated Hospital of \\ Medical College, Qing Dao University \\ P.R.China
}

\section{Introduction}

Hepatic masses constitute only $5 \%$ to $6 \%$ of all intra-abdominal masses in children (Pobeil \& Bisset, 1995) and primary hepatic neoplasms constitute only $0.5 \%$ to $2 \%$ of all pediatric malignancies (Davey \& Cohen, 1996). Primary hepatic neoplasms are the third most common abdominal malignancy in childhood, after Wilms' tumor and neuroblastoma (Davey \& Cohen, 1996). The majority of liver tumors in children are malignant. Only about one third of the liver tumors are benign (Jha et al., 2009).

Most children with benign or malignant liver masses present with a palpable mass on physical examination. Other presenting symptoms include pain, anorexia, jaundice, paraneoplastic syndromes, hemorrhage, or congestive heart failure. Although it is often obvious that these children have an upper abdominal mass, the organ of origin is often not clear without imaging.

Pediatricians and surgeons began to order more imaging studies because advances in imaging technology improved the diagnosis and management of disease. Imaging of pediatric hepatic masses has included multiple modalities, such as ultrasound, CT scan, MR imaging, angiography, and radionuclide techniques. Because surgical resection remains the mainstay of treatment for many of these lesions, detailed depiction of the extent of the mass and relationship to hepatic anatomy is essential. Ultrasound is usually the initial imaging modality in the evaluation of a child with a suspected abdominal mass. Ultrasound accurately excludes a mass when it is not present and identifies the organ of origin when a mass is present. Identifying the organ of origin helps determine the remainder of the child's imaging work-up. Ultrasound also evaluates whether a mass is cystic or solid and assesses vascular flow. When ultrasound confirms the lesion is in the liver, usually additional imaging is obtained with CT scan or MR imaging.

The advantage of CT requires less or no anesthesia due to faster scan times. So CT has always played a major role in the imaging of the liver. But at the same time pediatric patients present unique technical challenges for CT. Children are not simply small adults, and CT scan principles drawn from experience with adults can not accurately be extrapolated to the pediatric population. The methods of CT examination should be adjusted.

Whether CT scan or MR imaging is the modality of choice for definitive imaging of liver masses is a controversial issue. The choice is usually based on institutional experience and modality availability. Nevertheless, the development of multidetector row CT (MDCT) 
technology has helped CT to continue to excel in its already established indications (ie, hepatic lesion detection and characterization) and to add new clinical indications (ie, CT angiography for preprocedure mapping, liver perfusion). The fast pace of development challenged radiologists in terms of the cost of replacement of scanners, the optimization of CT protocols for existing indications, and the development of new protocols for the new applications introduced by the MDCT technology.

Hepatic tumors are classified into benign and malignant categories. Malignant hepatic neoplasms are twice as frequent as benign neoplasms and most of these are hepatoblastomas. Hepatocellular carcinoma, including the fibrolamellar variant, undifferentiated (embryonal) sarcoma, and angiosarcoma are less common malignant tumors. The common benign hepatic tumors are hemangioendothelioma, hemangioma, and mesenchymal hamartoma, with focal nodular hyperplasia and adenoma encountered less often (Kuhn et al., 2004; Helmberger et al., 1999; Emre \& McKenna, 2004; Pobiel \&, Bisset, 1995; Siegel et al., 2008).

\section{Techniques of CT scan in children liver}

Hepatic CT present significant technical challenges in children. There are several problems that are present in children but not present in adults. These include small patient size, lack of fat, and inability to suspend respiration or voluntary motion. With proper attention to technique, these problems can be minimized and can improve diagnostic quality .

The introduction of helical CT has greatly improved the capabilities of CT scanning. The subsecond scanning techniques eliminate respiratory misregistration, decrease the need for sedation, and enable scanning during arterial and venous phases of contrast administration. The helical data set also enables threedimensional imaging.

\subsection{Preparation of children before the examination}

To acquire an optimal CT examination adequate prescan patient preparation is as important as the optimization of the CT technique. Issues that should be addressed are (1) psychological preparation of children and parents (including the scanner environment), (2) the need for sedation or general anaesthesia, (3) oral contrast material preparation, and (4) intravenous (IV) contrast material preparation.

\subsubsection{Psychological preparation before CT scanning}

Adequate prescan patient preparation should include: (1)Age- and intellect-adapted information to the child and information for the parents about the CT examination (including written information, simulation, coaching or other forms of playing therapy). (2)Inviting one or both parents to stay with the child before, during and after the investigation.(3)Adaptation of the scanner environment to children (for instance by using a painted curtain covering the CT gantry, and (projection of) paintings on the wall or ceiling). This will help to reduce the anxiety of the child and positively influence their mood, increasing the success rate of the CT examination without the need for sedation or general anaesthesia.

\subsubsection{Sedation}

There has been a phenomenal increase in the number of diagnostic radiology procedures in the past decade. Consequently, the demand for fast, effective and safe sedation for children 
has grown exponentially as well. The parents expect an anxiety free experience for their children. The radiologists expect the child to be cooperative and not move (Krauss \& Green, 2000; Shankar, 2008). In general, children 5 years of age and older will be able to undergo a CT examination without sedation after thorough patient instruction. However, there are still situations in which sedation or general anaesthesia will be required depending on the type of investigation, age and mental ability of the child and the clinical situation and question. The way sedation and general anaesthesia is organised depends largely on local agreements and legislations. The drugs most frequently used for sedation are oral chloral hydrate and intravenous pentobarbital sodium (American Society of Anesthesiologists Task Force,1996; Bisset \& Ball,1991; Committee on Drugs,1992; Cote,1994; Frush et al,1996; Pereira et al,1993; Strain, 1988; Siegel, 1999; Nievelstein,2010). Oral chloral hydrate, 50 to $100 \mathrm{mg} / \mathrm{kg}$, with a maximum dosage of $2000 \mathrm{mg}$, is the drug of choice for children younger than 18 months. Intravenous pentobarbital sodium, $6 \mathrm{mg} / \mathrm{kg}$ with a maximum dose of $200 \mathrm{mg}$, is advocated in children older than 18 months. It is injected slowly in fractions of one fourth the total dose and is titrated against the patient's response. This is an effective form of sedation with a failure rate of less than $5 \%$.

Fentanyl citrate is in the class of narcotics and has the combined benefits of sedation and analgesia. Fentanyl is given for pain control. It is administered intravenously in a dose of 1.0 $\mu \mathrm{g} / \mathrm{kg}$. The drug is given slowly over $5 \mathrm{~min}$. Maximum cumulative dose is $3 \mu \mathrm{g} / \mathrm{kg}$. For those patients weighing over $25 \mathrm{~kg}$, fentanyl is given in $25-\mu \mathrm{g}$ aliquots until the desired analgesia is achieved. Onset is almost immediate, and duration of action is 30 to $60 \mathrm{~min}$.

Regardless of the choice of drug, the use of parenteral sedation requires the facility and ability to resuscitate and maintain adequate cardiorespiratory support during and after the examination. After being sedated, the infant or child is placed on a blanket on the CT table. For the CT examination, the arms routinely are extended above the head to avoid streak artifacts and to provide an easily accessible route for intravenous injection. The upper arms can be restrained with sandbags, adhesive tape, or Velcro straps.

Patients who are to receive parenteral sedation should have no liquids by mouth for 3 hours and no solid foods for 6 hours prior to their examination.

\subsubsection{Oral contrast material}

As small children lack large amounts of intraabdominal fatty tissue, it is more difficult to interpretation of a CT of the abdomen in children than in adults. That is why we prefer US as the imaging modality of first choice for abdominal clinical problems in childhood. If CT of the abdomen is indicated, adequate oral contrast intake is often essential for the evaluation. Opacification of the small and large bowel is needed to determine the extent of extrahepatic disease (Siegel, 1998; Siegel, 1999; Siegel, 2001).A dilute (1-2\%) solution of water-soluble, iodine-based oral contrast agent is given by mouth or through a nasogastric tube if necessary. A non-ionic iodinated oral contrast agent is often preferred because of the risk of aspiration. The oral contrast agent can be mixed with fruit juice if needed to mask the unpleasant taste. The gastrointestinal tract from the stomach to the terminal ileum usually can be well opacified if the contrast agent is given in two volumes, one is 45 to $60 \mathrm{~min}$ before the examination and the other is $15 \mathrm{~min}$ prior to scanning. The first volume should approximate that of an average feeding. The second volume should be approximately one half that of the first (Table 1). 


\begin{tabular}{ccc}
\hline Age & $\begin{array}{c}\text { 1st dose }(\mathrm{ml}) \\
( \pm 1 \mathrm{~h} \text { prior to CT })\end{array}$ & $\begin{array}{c}\text { 2nd dose }(\mathrm{ml}) \\
(15 \mathrm{~min} \text { prior to CT })\end{array}$ \\
\hline 1-6 months & $90-120$ & $45-60$ \\
6 months-1 year & $120-180$ & $60-90$ \\
1-4 years & $180-270$ & $90-135$ \\
4-8 years & $270-360$ & $135-180$ \\
8-12 years & $360-480$ & $180-240$ \\
12-16 years & $480-600$ & $240-300$ \\
\hline
\end{tabular}

Table 1. Age-based amounts of oral CM for a biphasic preparation protocol, $1 \mathrm{~h}$ and $15 \mathrm{~min}$ prior to CT examination (Frush, 2008; Siegel, 2008; Nievelstein, 2010)

\subsection{Intravenous contrast material}

For the administration of intravenous (IV) contrast material, the use of a power injector instead of hand injection is preferred. Contrast is administered by a power injector if a 22gauge or larger plastic cannula can be placed into an antecubital vein. (Siegel,1999a; Siegel,1999b; Roche,1996; Siegel,2001) The injection rate is determined by the caliber of the intravenous catheter. Contrast material is infused at $1.2 \mathrm{~mL} / \mathrm{sec}$ for a 22 -gauge catheter, at $1.5 \mathrm{~mL} / \mathrm{sec}$ for a 20-gauge catheter, and at $2 \mathrm{~mL} / \mathrm{sec}$ for an 18-gauge needle. A hand injection is needed if intravenous access is through a peripheral access line, a smaller caliber antecubital catheter or butterfly needle, or a central venous catheter.

The standard contrast material used for IV administration is a non-ionic, low-osmolar contrast agent with a concentration between 240 and $400 \mathrm{mg} \mathrm{I} / \mathrm{ml}$ (most frequently $300 \mathrm{mg}$ $\mathrm{I} / \mathrm{ml}$ ). Adjusting the iodine dose for body weight is particularly crucial in children because of the wide range of body sizes. The traditional dose of contrast medium administered in children is $2 \mathrm{~mL} / \mathrm{kg}$, with a maximum dose of $150 \mathrm{~mL}$ (Frush,1997). This scheme, which was based on use of low-concentration contrast media (240-300 mg I/mL), has been commonly practiced since early CT more than 30 years ago and is widely used even in the current era of fast MDCT and the general use of higher-concentration contrast media. Short scanning times offered by fast MDCT allow improved contrast enhancement and more efficient use of contrast media(Bae \& Heiken,2005; Bae,2007; Bae ,2008).Some scholar indicated that to achieve consistent aortic or hepatic contrast enhancement in pediatric patients with abdominal 64-MDCT, the amount of contrast medium should be adjusted to the patient's body weight for all ages of pediatric patients: approximately $1.5 \mathrm{~mL} / \mathrm{kg}$, or $0.525 \mathrm{~g} \mathrm{I} / \mathrm{kg}$, to yield $116 \mathrm{HU}$ of hepatic attenuation or 50-55 HU of hepatic enhancement. (Bae ,2008)

In children the circulation time varies widely which makes adequate scan timing more difficult. Furthermore, the size, position and type of cannula will differ among different age groups, with as a consequence varying injection rates. In general, an injection rate of 2.0 $\mathrm{ml} / \mathrm{s}$ suffices for most paediatric indications, especially when younger than 12 years of age. An empirically determined fixed delay time usually suffices for most routine indications, especially in the younger age group. However, the routine use of bolus tracking techniques is strongly recommended for most body indications, especially in case of a CT angiography (CTA) or arterial-phase CT (Bae \& Heiken, 2005). An alternative for scan timing is the test bolus technique, although this technique is not suitable in very small children as the total volume of contrast material available is often too small. Both techniques share the disadvantage of additional (monitoring) scans, increasing the radiation dose for the child. 
This additional dose should be weighed against the benefit of improved and individualized scan timing, and when applied the monitor scans should be obtained with a low-dose technique to limit this additional radiation dose.

Patients who are to receive intravenous contrast medium for the CT examination should be NPO (nothing per mouth) for 3 hours to minimize the likelihood of nausea or vomiting with possible aspiration during a bolus injection of intravenous contrast medium.

\subsection{Selective organ shielding}

The use of bismuth shielding of radiosensitive organs (e.g., breast, thyroid gland and eye lens) to reduce organ doses has been suggested. However, these shields may also reduce the amount of radiation reaching the detector ring in some projections and may add noise or artifacts to the images, especially if no standoff pads are used (Vollmar \& Kalender, 2008). A fundamental study by Geleijns et al.(Geleijns et al,2006) showed that the reduction in organ dose can also be achieved more efficiently by lowering the tube current. In addition, these shields may complicate the use of dose modulation techniques with the risk of increasing radiation dose to the child. Therefore, it remains to be seen if selective organ shielding will be of any additional benefit if the CT protocols are already maximally optimised for children (Vollmar \& Kalender, 2008; Geleijns et al., 2006; Leswick et al., 2008). We will therefore not advocate this method.

\subsection{Scan and technical parameters}

The CT protocol for evaluation of a possible liver mass is performed with a dual-phase spiral CT scan through the liver, with additional delayed scans obtained as needed (Aytekin et al,2005).A slice thickness of $5 \mathrm{~mm}$ is used with a pitch of 1 to 1.5, depending on patient size, and 3-mm reconstructions. For dual-phase imaging, the arterial dominant phase of liver enhancement should be initiated at 10 to $15 \mathrm{sec}$ after the start of the contrast bolus. The portal venous phase is initiated as soon as possible after completion of the arterial phase of enhancement (interscan delay usually between 20 and $40 \mathrm{sec}$ ). In order to minimise the radiation dose to the child, some experts suggests that an empirically determined fixeddelay time of $50 \mathrm{~s}$ after initiation of the IV injection of contrast material usually suffices, resulting in a CT examination during the portal venous phase (Roebuck, 2009).

The scan field of view (FOV) should be tailored as much as possible to the size of the body region of interest. The major advantage of a smaller scan FOV is the higher spatial resolution, as the pixel size decreases with smaller FOV. The effect of the display FOV on resolution is often different-while some increase in resolution may result, after a certain threshold pixels are only blown up.

Due to the smaller size of children it is usually possible to lower the tube voltage with maintenance or even improvement of the diagnostic image quality and resulting in a significant dose reduction. In most children a tube voltage of $80-100 \mathrm{kVp}$ will suffice, especially in children with a body weight $<45 \mathrm{~kg}$. In adolescents, a tube voltage of $120 \mathrm{kVp}$ for the abdomen is usually sufficient. The tube current (mA) should be adapted to the size or weight of the child. Most modern MDCT scanners have tube rotation times between 0.3 and $0.5 \mathrm{~s}$ resulting in shorter scan times. In terms of image quality a rotation time of $0.5 \mathrm{~s}$ is often the best option. The tube current modulation techniques, available on almost all modern MDCT scanners, are increasingly used in paediatric MDCT (Nievelstein, 2010). 


\subsection{Techniques of CT reconstruction}

State-of-the-art cross-sectional imaging techniques allow radiologists to visualize disease with greater certainty by subtracting the impact of overlying tissues, thus allowing separate evaluation of individual organs, which aids in the detection and characterization of pathology.

Radiologists discovered in the late 1970s that although diagnosis based on axial CT images alone was more sophisticated than with plain radiography, the lack of a third dimension (e.g. sagittal and coronal dimensions) was frequently frustrating. Many referring physicians with no basic training in cross-sectional imaging still encounter difficulties in appreciating normal anatomy and pathology on transverse CT images, being more familiar with anatomy depicted in the coronal plane.

Following the somewhat crude three-dimensional computer rendering algorithms initially developed in late 1970s, that allowed formation of images in the third dimension from data acquired in the axial plane(Fig.1d), the development of single-slice helical CT and more recently multidetector CT (MDCT) scanners has opened new chapters in 3D imaging. These advances were made possible by the rapid acquisition of volumetric data in the lower case z-axis using thin slices and improved rendering algorithms, which facilitate exquisite 3D reformats, devoid of degradation by respiration and other physiological movements. The pace of progress is being hastened with the rapid developments in MDCT technology (Aytekin et al, 2005; Maher, 2004). We describe 3D rendering techniques available for abdominal imaging including multiplanar reconstructions, surface rendering, virtual endoscopy, volume rendering and maximum intensity projections. These 3D reformats can show the feeding arteries and venous drainage of detected lesions better, In addition, they are preferred by surgeons for preoperative planning, because the relationship of the lesions to the blood vessels and bile ducts is better delineated (Sahani et al,2002)(Fig.cd). Multiplanar volume rendering and creation of maximum intensity projections from MDCT data allow evaluation of both parenchymal and vascular detail in real time, interactively.

Multiplanar reconstruction (MPR) provides efficient computation of images that lie along the non-acquired orthogonal orientations of the scanned volume by readdressing the order of voxels in the scanned volume. It is a fast and interactive algorithm that can represent several arbitrary planes at once and create multiplanar display in real-time. Generally, it is helpful whenever pathology cannot be accurately assessed on axial plane images alone(Fig.1 a).

Volume rendering (multiplanar volume rendering, MPVR) is the visualization and manipulation of objects represented as sampled data in three or more dimensions. The technique interpolates the entire data set rather than editing a single scan to generate $3 \mathrm{D}$ images directly from scanned volume data. Unlike other projection techniques such as SSD and MIP, MPVR does not distort objects in the reconstructed planes. It allows "quick view" of large MDCT scan data sets with comprehensive details of the anatomic orientation of lesion or structures of interest.

The maximum intensity projection (MIP) technique displays the pixels of greatest intensity along a predefined axis of the image. It is useful for the depiction of vascular anatomy when there is a large difference between attenuation values (Hounsfield value, $H U$ ) of vessels opacified by contrast agent, and the surrounding tissues(Fig.1b). MIP is useful for all types of CT angiography and has also been used for CT Urography (Caoili et al, 2002)(Cody,2002). 


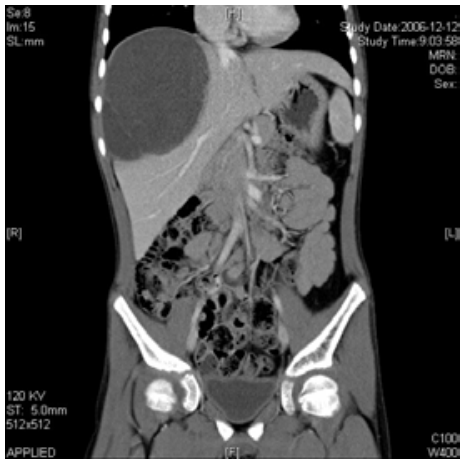

(a)

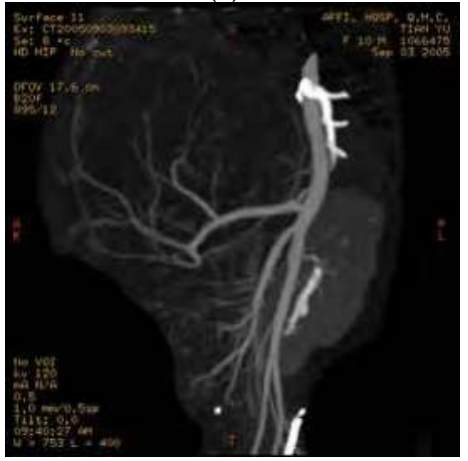

(c)

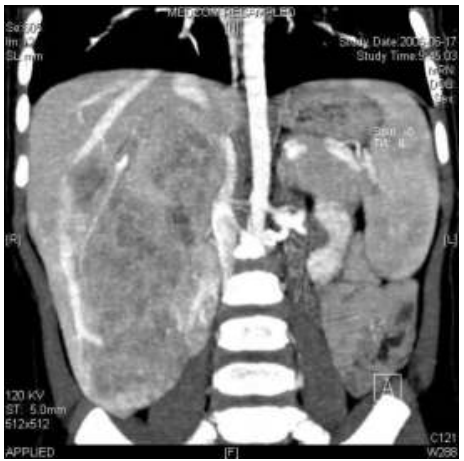

(b)

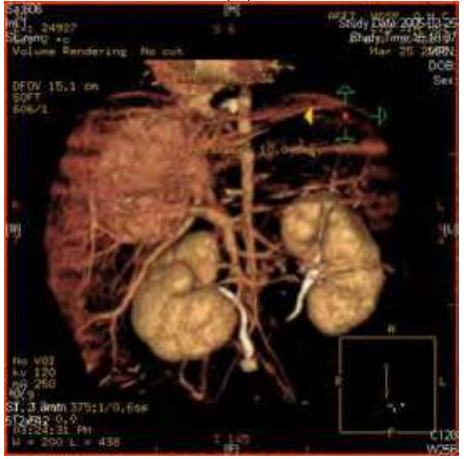

(d)

Fig. 1. a Coronal multiplanar reconstruction (MPR);b Maximum intensity projection (MIP) shows the elationship between the tumor and vein;c CT angiography(CTA); $\mathrm{d} 3 \mathrm{D}$ imaging.

\section{Pathological characteristics and radiological features of primary liver tumors in children}

The differential diagnosis for liver tumors in children includes benign and malignant neoplasms. Malignant neoplasms are usually hepatoblastomas or hepatocellular carcinomas (HCC) (Siegel,2001; Scuza \& Narla,1992).Benign lesions are usually hemangioendotheliomas and less commonly mesenchymal hamartoma, cavernous hemangioma, focal nodular hyperplasia, and hepatic adenoma (Siegel,2001; Scuza \& Narla,1992).Clinical information plays an important role in narrowing the differential diagnosis in cases where the imaging findings are nonspecific. Hemangioendothelioma is the most common mass in the first 6 months of life. Hepatoblastoma, mesenchymal hamartoma usually present in the first 3 years of life. HCC, focal nodular hyperplasia, and hepatic adenoma tend to occur in older children and adolescents. Certain liver tumors, such as hepatoblastoma and HCC, are associated with elevated serum alphafetoprotein levels (Greenberg \& Filler, 1997; Siegel,2001).The clinical presentation also can suggest a specific diagnosis. Congestive heart failure in a neonate with a liver mass suggests the diagnosis of hemangioendothelioma. (Donnelly \& Bisset, 1998) The role of imaging is to determine the organ of origin, character, and extent of the lesion. (Powers et al., 1994) 


\subsection{Malignant tumors \\ 3.1.1 Hepatoblastoma}

Hepatoblastomas are the most common primary liver tumors of the children with a peak presentation at 1-2 years of age and a male:female ratio of 2:1(Kuhn et al,2004; Helmberger et al,1999; Jha et al,2009). Less frequently, hepatoblastomas may also occur in older children, up to 15 years of age. Histologically, HB can be classified into an (a) epithelial, (b) mixed (epithelial/mesenchymal), or (c) anaplastic type. Epithelial HB is the most common type $(60 \%)$. all HBs are large with an average diameter at diagnosis of $10 \pm 12 \mathrm{~cm}$. Unenhanced CT typically shows a relatively well-defined, heterogeneous mass, slightly hypodense compared with liver tissue, with or without calcifications(Fig.2). On contrast-enhanced CT, the tumor reveals a heterogeneous enhancement (Fig. 3), The tumor enhances during the hepatic arterial phase of dynamic contrastenhanced CT and becomes hypoattenuating in the portal venous phase of enhancement. The tumor thrombus can invade the portal vein , spread along inferior vena cava(IVC) and encroach in the lumen of right atrium. Metastasis may be seen in lymph nodes and lung parenchyma, rarely in the bones and brain.

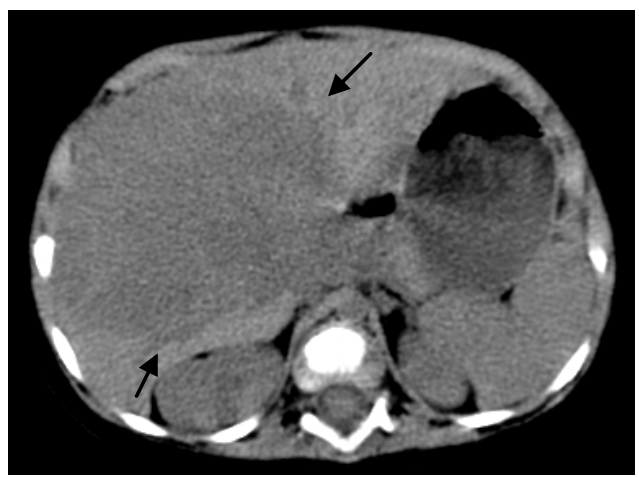

Fig. 2. A 5-year-old boy with hepatoblastoma. Unenhanced CT shows a relatively welldefined mass, slightly hypodense compared with liver tissue (arrowheads).

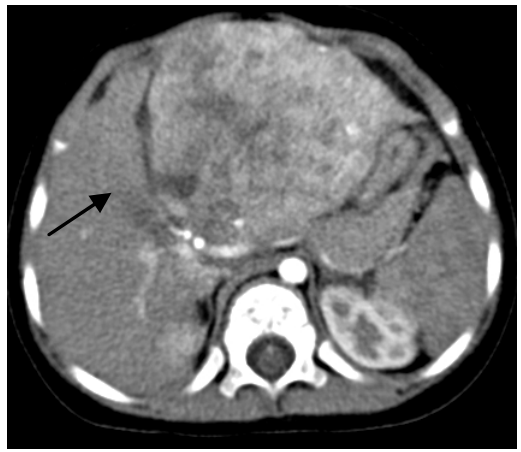

(a)

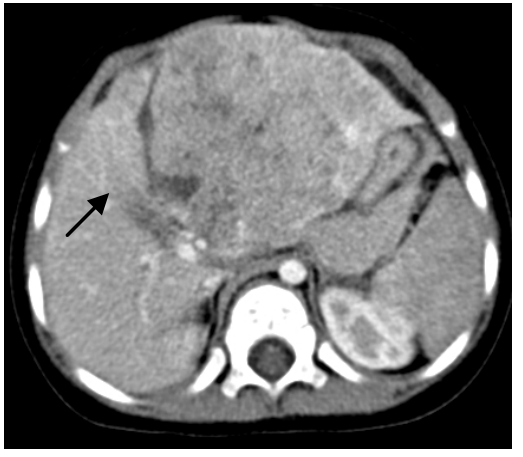

(b)

Fig. 3. A 2-year-old boy with hepatoblastoma. Axial enhanced CT images of the abdomen demonstrate an ill-defined heterogeneous mass (arrowheads) with areas of necrosis appearing hypodense to the liver parenchyma 


\subsubsection{Hepatocellular carcinoma (HCC)}

HCC is the second most common pediatric liver malignancy after hepatoblastoma. In the pediatric population, HCC has a median age of 12 years, with a range of 5 to 15 years, and is rare under 5 years (Greenberg \& Filler, 1997;Davey \& Cohen, 1996;Siegel, 2001). On CT, HCCs present with highly variable and non-characteristic features: the tumors may be homogeneous or heterogeneous, solitary or multifocal, well- or ill-defined. HCCs are typically isodense or slightly hypodense compared with liver parenchyma on unenhanced CT images and show an early arterial contrast enhancement and a rapid wash-out on enhanced CT (Fig. 4). Invasion of portal veins, hepatic veins, hepatic arteries and inferior vena cava may be seen. The diagnosis of an underlying cirrhosis may help in the differential diagnosis, but is rare in children. Diffuse involvement of the liver leads to a diffusely hypodense liver on CT. HCCs metastasize to lung, bone, skin and brain.

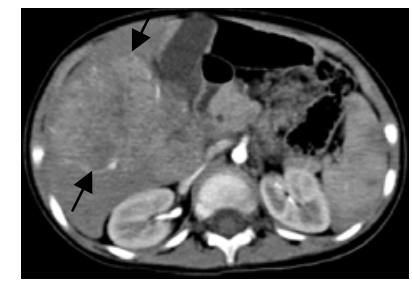

(a) Arterial phase

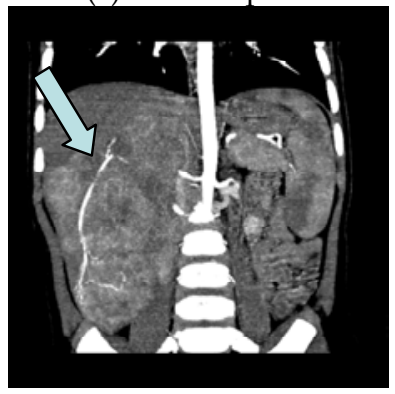

(d) MIP of Arterial phase

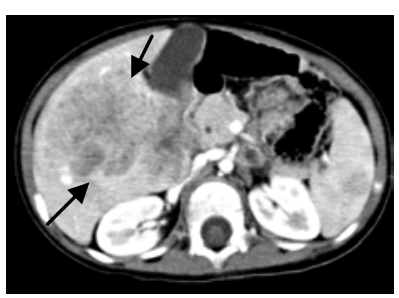

(b) Portal venous phase

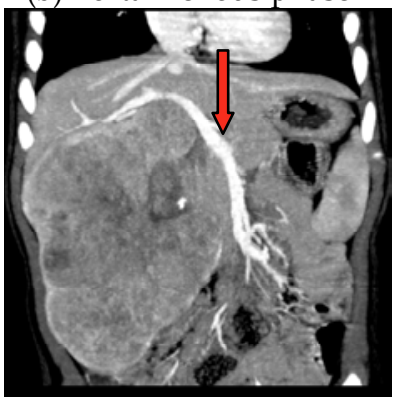

(e) MIP of Venous phase

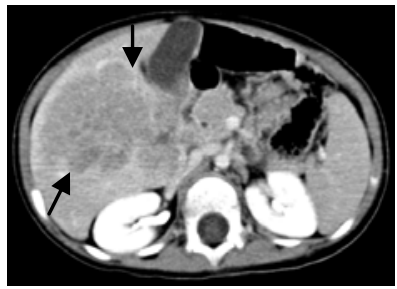

(c)Delayed phase

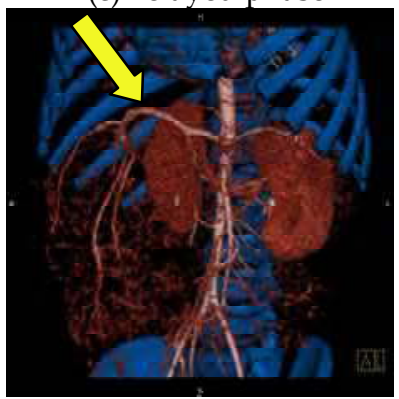

(f) Volume rendering(VR)

Fig. 4. A 2-year-old boy with HCC. Enhanced CT images of the abdomen shows an illdefined heterogeneous mass (black arrowheads) with early arterial contrast enhancement and a rapid wash-out on portal venous phase and delayed phase $(a 、 b 、 c) ; d$. Maximum intensity projection reconstructed from the axial MDCT images obtained during the early arterial phase of contrast enhancement demonstrates artery in tumor (blue arrowheads); $\mathrm{e}$. Maximum intensity projection obtained during the portal venous phase of contrast enhancement shows the relationship between tumor and portal veins (red arrowheads) ;f. Volume rendering(VR) shows the feeding arteries of the tumor (yellow arrowheads)

\subsection{Benign tumors}

\subsubsection{Infantile hepatic hemangioma or hemangioendothelioma}

Hepatic hemangiomas and hemangioendotheliomas are the most common vascular hepatic tumors in the first year of life (50\% of the benign tumors) (Kuhn et al,2004, Helmberger et al,1999, Jha et al,2009). Most affected patients are young infants less than 6 months old 
(85\%), the male:female ratio being 1:2. The two lesions show distinct histopathological characteristics. Infantile hepatic hemangiomas are benign vascular lesions. Epitheliod hemangioendotheliomas are also primarily benign, endothelium lined vascular masses, but may show a malignant potential (Emre \& McKenna, 2004). In the proliferative phase, there is characteristic hypercellularity, endothelial proliferation and dilatation of vascular spaces, leading to a characteristic 'cavernous appearance'. On unenhanced computed tomography (CT), hemangiomas and hemangioendotheliomas have a lower attenuation than the liver parenchyma with occasional hemorrhage(Fig. 5a). Calcifications may be seen in up to $40 \%$ of cases. On contrast-enhanced CT there is a characteristic intense, nodular peripheral rim enhancement with central progression (Fig. 5b,c,d). Central filling defects may occur in larger lesions due to central thrombosis or fibrosis. On delayed enhanced images, infantile hemangiomas and hemangioendotheliomas show a characteristic persistent enhancement, a distinct feature compared with other liver tumors.

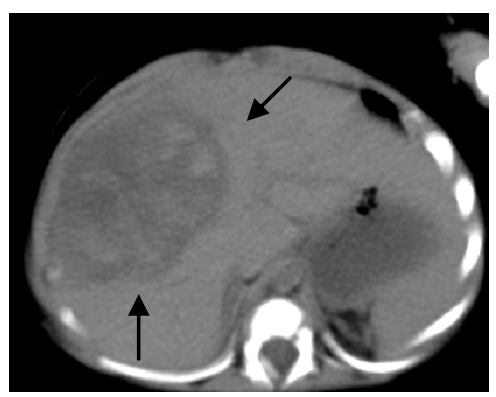

(a) Unenhanced CT

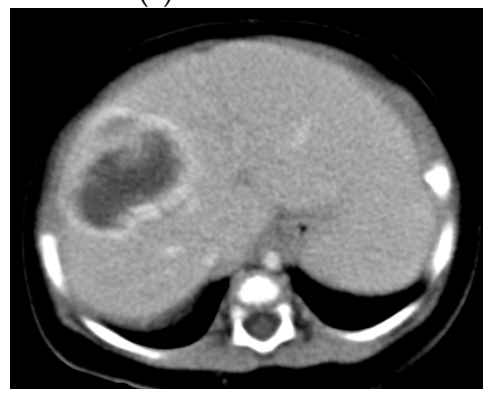

(c) Portal venous phase

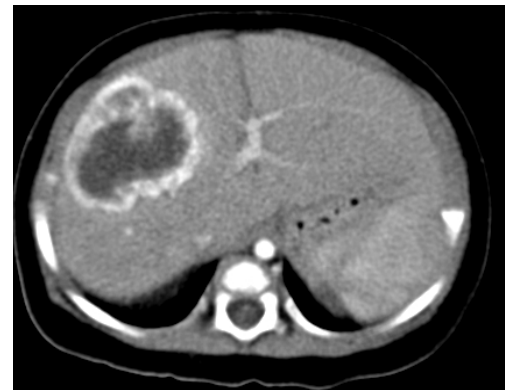

(b) Arterial phase

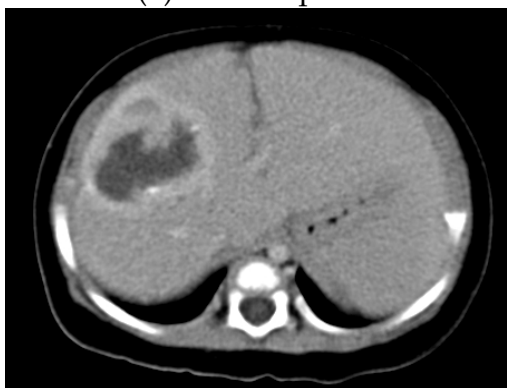

(d) Delayed phase

Fig. 5. A 10-month-old girl with hemangioedothelioma . a Unenhanced axial CT slice with soft tissue window. The tumor (arrowheads) is seen as a well-defined, lobulated, low attenuation mass in the right lobe of the liver. Hyperintensity in the center of the lesion represents dystrophic calcification. b、c、d Contrast-enhanced CT showsr peripheral rim enhancement and persistent enhancement on delayed phase.

\subsubsection{Mesenchymal hamartoma}

It is the second most common benign hepatic lesion in the perinatal period. It is seen usually in children less than 2 years of age with a male:female ratio of 2:1. The tumor arises from mesenchymal tissue around the portal tract. Grossly, the lesion is not encapsulated and is 
typically composed of multiple cysts, filled with clear or mucoid fluid. (Stringer \& Alizai, 2005; Chang et al,2006). CT images show a multilocular low-attenuation cystic mass with enhancing septae and stroma (Fig. 6). Calcification is generally not seen.

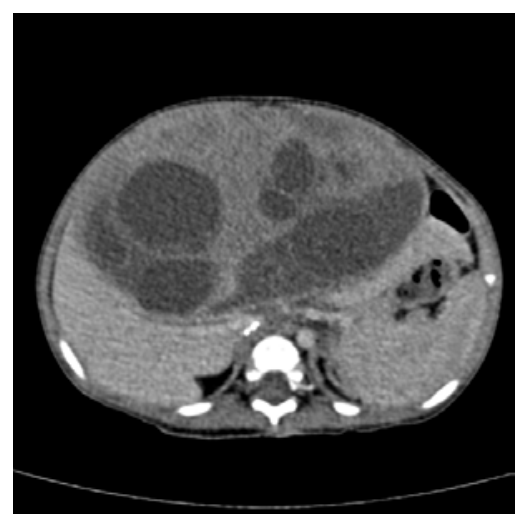

(a) Enhanced axial CT of Portal venous phase

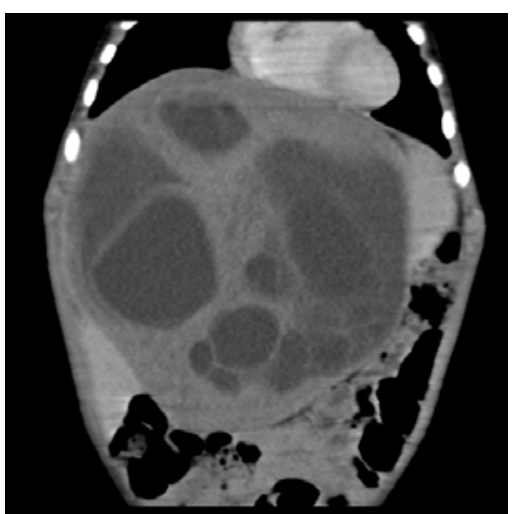

(b) Coronal reformats

Fig. 6. Huge mesenchymal hamartoma in a 12-month-old boy. a Enhanced axial CT shows multilocular low-attenuation cystic mass with enhancing septae and stroma. b On coronal reformats the mass was noted to occupy large areas in the abdomen

\subsubsection{Hepatic adenomas}

Adenomas are rare in the pediatric population. They are usually seen in teenagers with a female preponderance. They may be seen in patients with glycogenstorage diseases. Histologically, adenomas present as encapsulated, rounded masses, which consist of hepatocytes, Kupffer cells, rudimentary portal tracts and distorted biliary elements. The Kupffer cells in adenomas have a decreased or absent phagocytic activity, This is a distinctive feature compared with FNHs. CT demonstrates an isodense or slightly hypodense mass with a well-defined border due to the presence of a capsule. Hemorrhage may lead to a heterogeneous appearance. Adenomas typically show an early arterial enhancement followed by a rapid wash-out on enhanced CT.(Fig.7)

\section{The value of CT scan in guiding the surgical treatment}

For most hepatic malignancies, complete tumor resection or liver transplantation is essential for cure. Types of liver resection performed include left lobectomy; left lateral segmentectomy; right lobectomy; or trisegmentectomy (right lobe and medial segment of the left lobe). Therefore, a mass must be confined to the left or right lobe or the right lobe plus the medial segment of the left lobe to be considered resectable. If a lesion does not meet anatomic requirements for resectability at initial imaging, the child is often initially treated with chemotherapy, with or without radiation, and then re-imaged. Therefore, proper imaging of the liver is necessary and can shorten the surgical duration and increase the accuracy of the resection (Kinoshita et al.,2009; Dong et al.,2007)

The major role of liver imaging is to define accurately the extent of the lesion in relation to hepatic lobar anatomy and vascular and biliary structures for preoperative planning and to 


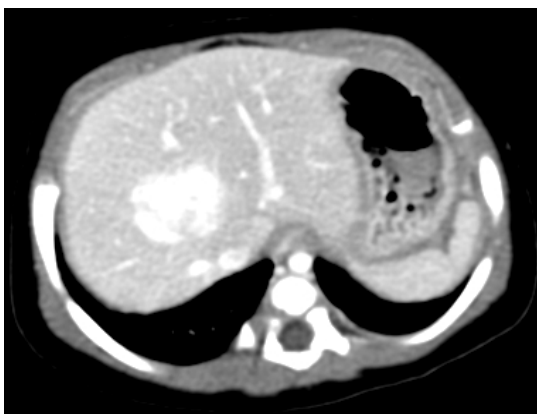

(a) Arterial phase

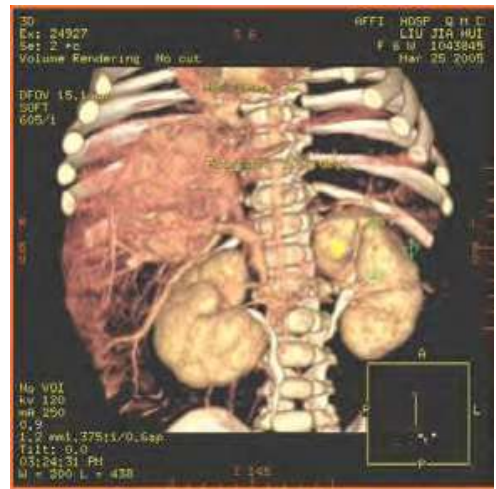

(c) Volume rendering(VR)

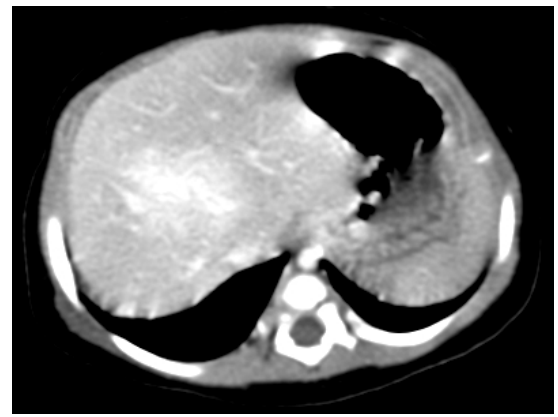

(b) Portal venous phase

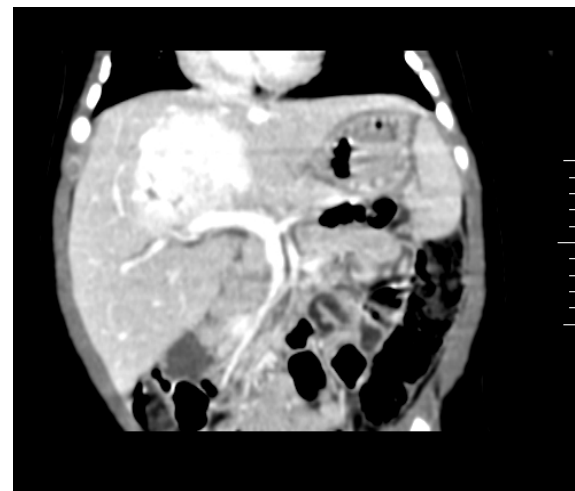

(d)Coronal reformats

Fig. 7. A 3-year-old girl with hepatic adenomas Contrasted CT scans show an early arterial enhancement followed by a rapid wash-out on enhanced CT.

monitor tumor response to chemotherapy or radiation. Worldwide, computed tomography (CT) is undoubtedly the most frequently used diagnostic tool in the radiologist's armamentarium for studying the liver. The development and rapid clinical acceptance of single-detector helical (spiral) computed tomography (HCT) during the last decade and, more recently, the introduction of multidetector CT (MDCT) have resulted in significantly improvements of the ability to study the liver. In addition to technical advances, such as shorter scanning times, multiplanar imaging, and improved ability to perform multiphasic contrast-enhanced studies, newer and better intravenous contrast media and advances in postacquisition data processing techniques have renewed the enthusiasm for using hepatic CT scanning(Kinoshita et al.,2009;Frericks et al.,2004).(Fig.8)

Before the application of the three-dimensional imaging with spiral CT, angiography was the most common imaging approach to determine tumor location and relation to blood vessels. However, the invasiveness and the need for anesthesia limit the use of the approach. Although these scans may roughly determine the size and location of the tumor, the precise location of the tumor in relation to crucial blood vessels cannot always be accurately assessed. Therefore, the surgeon relies heavily upon surgical exploration; because one-stage resection cannot be guaranteed, some parents decline the treatment. 


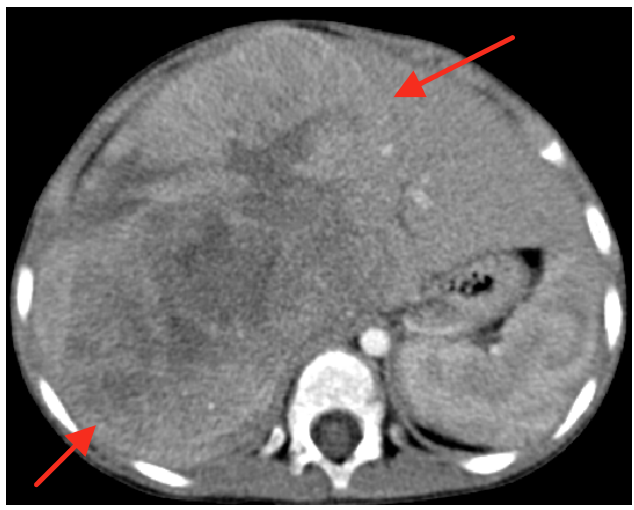

(a) Enhanced axial CT-Arterial phase

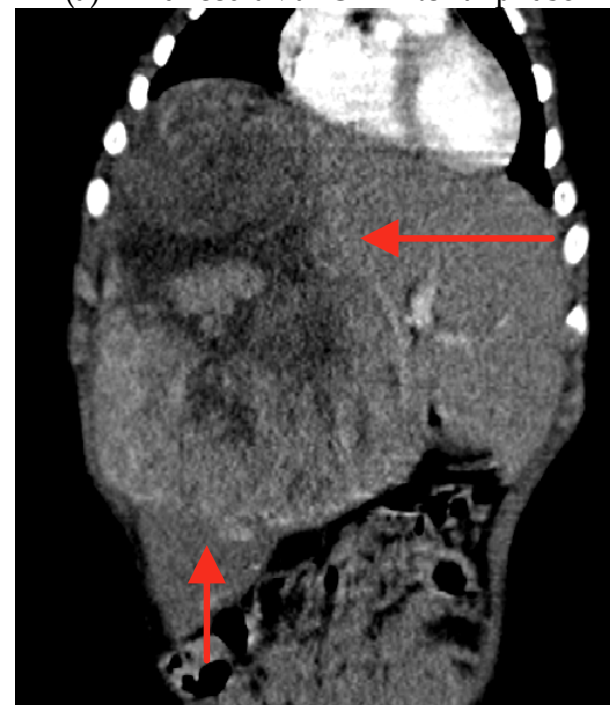

(c) Coronal reformat

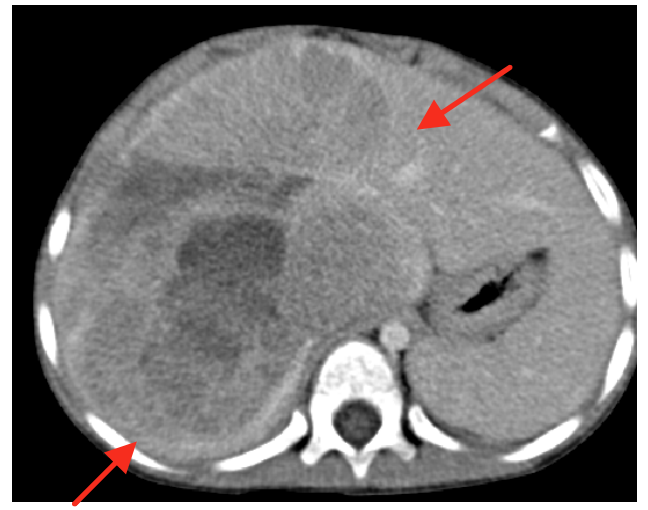

(b) Enhanced axial CT-Portal venous phase

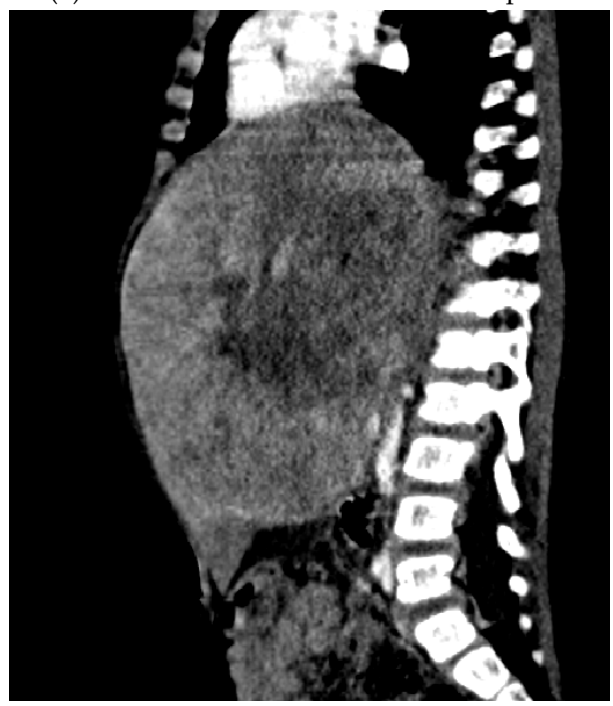

(d) Sagittal reformat

Fig. 8. Multiplanar imagings can show the extent of the lesion accurately(red arrowheads).

MDCT makes it possible to image the precise vascular anatomy including the anomalous branches, feeding arteries, or drainage veins (Kinoshita et al.,2009; Dong et al.,2007). the reconstructed images from MDCT were never inferior to those obtained by angiography. Therefore, when a chemoembolization by TACE is not necessary, this MDCT reconstruction is considered to provide a sufficient evaluation of the vessels. On the other hand, each image phase (arterial phase, equilibrium phase, portal phase) could be independently and simultaneously extracted or combined, respectively (Nievelstein RA et al.,2010). (Fig.9) (Fig.10) (Fig.11)

Furthermore, the software program for volumetry provides a proposed remnant liver volume and an optimal cut line of the liver. Various preoperative simulations can thus be considered. This volumetric analysis positively contributes to the safety of the procedure by assisting in the selection of the optimal operations. 


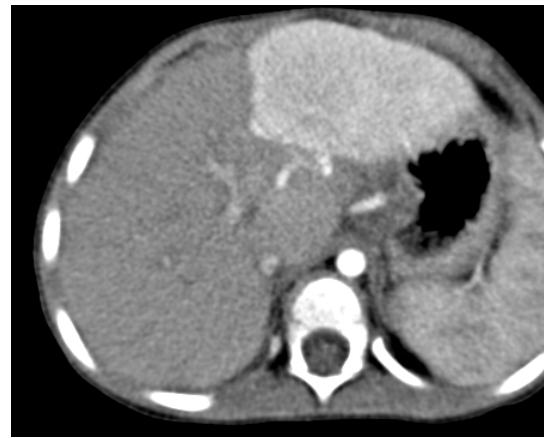

(a) Enhanced axial CT-Arterial phase

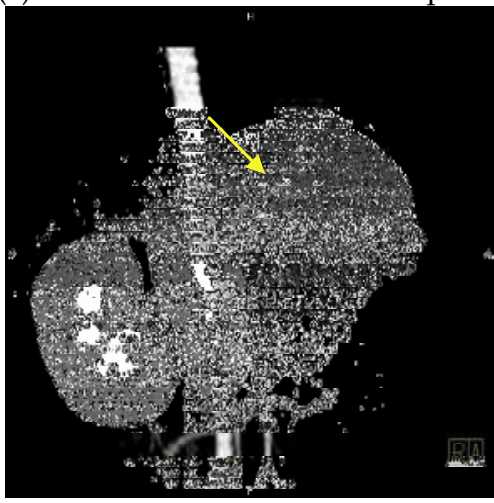

(c) Three-dimensional imaging

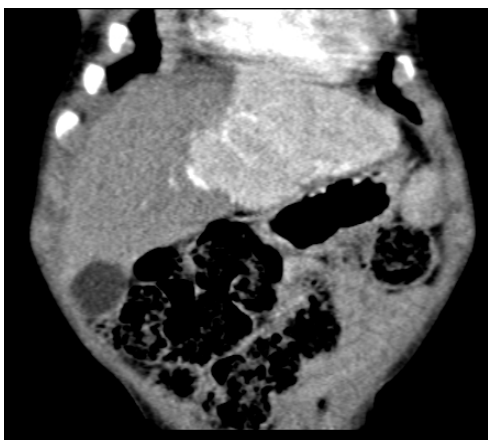

(b) Coronal reformat

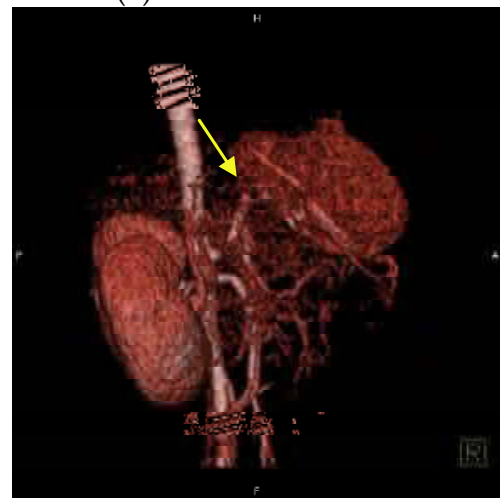

(d) Three-dimensional imaging

Fig. 9. Multiplanar imagings can show the extent of the lesion accurately $(a, b)$; The feeding artery of the tumor and its anomalous branches are detected by 3-D reconstruction images(yellow arrowheads). (c、d).

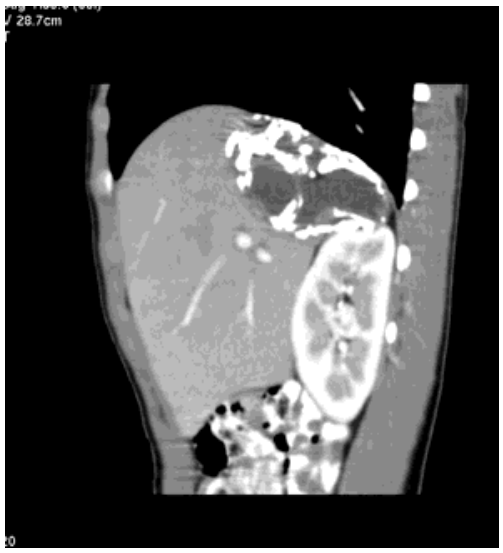

(a) Sagittal reformat

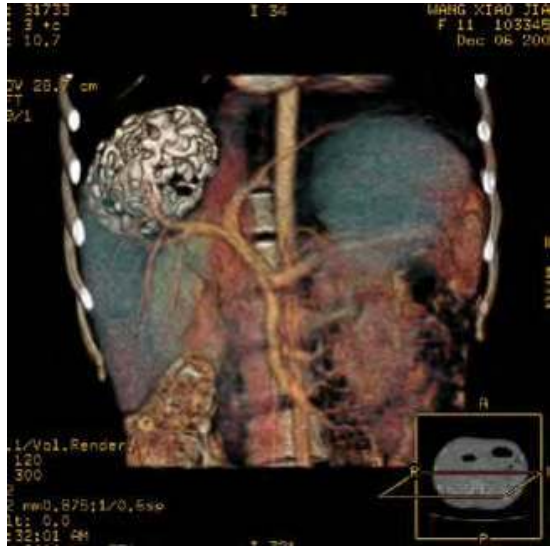

(b) Three-dimensional imaging

Fig. 10. Multiplanar imaging and three-dimensional imagingshow the tumor very clear. 


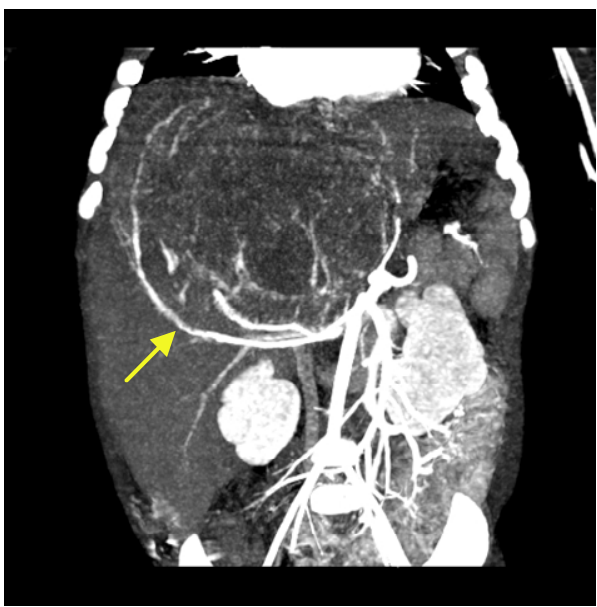

(a)

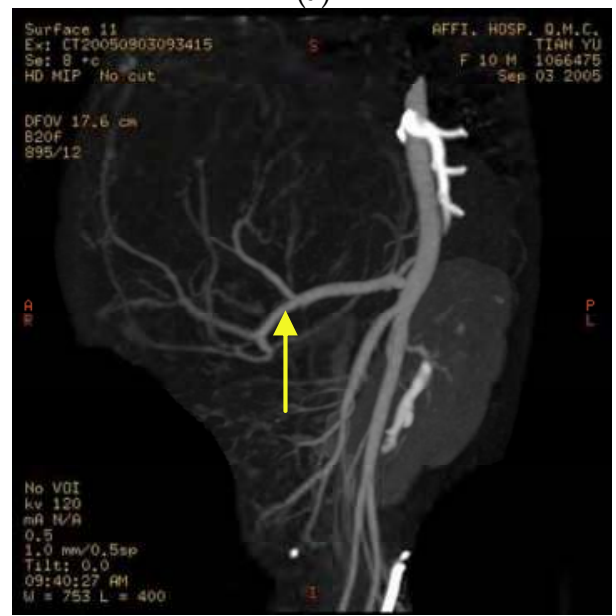

(c)

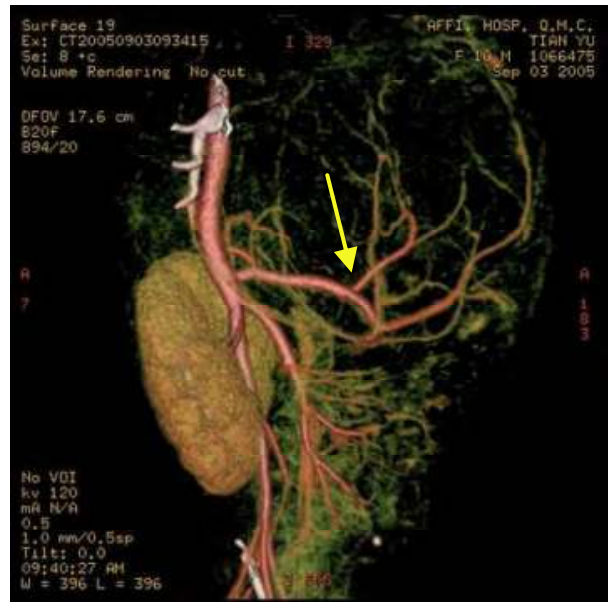

(b)

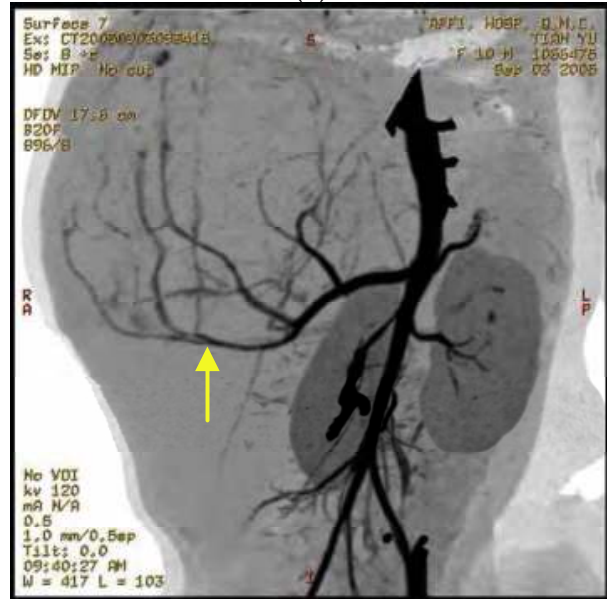

(d)

Fig. 11. CT angiography shows the feeding artery of the tumor and its anomalous branches(yellow arrowheads).

\section{Clinical applications of CT 3-D reconstruction imaging for diagnosis and surgery in children with large liver tumors or tumors at the hepatic hilum}

Multidetector CT (MDCT) scanners has opened new chapters in 3D imaging. These advances were made possible by the rapid acquisition of volumetric data in the lower case z-axis using thin slices and improved rendering algorithms, which facilitate exquisite 3D reformats, devoid of degradation by respiration and other physiological movements. The pace of progress is being hastened with the rapid developments in MDCT technology.

Curing the patients with liver tumor and prolong survival are current principles for surgeon, complete tumor resection without liver dysfunction is essential, especially for 


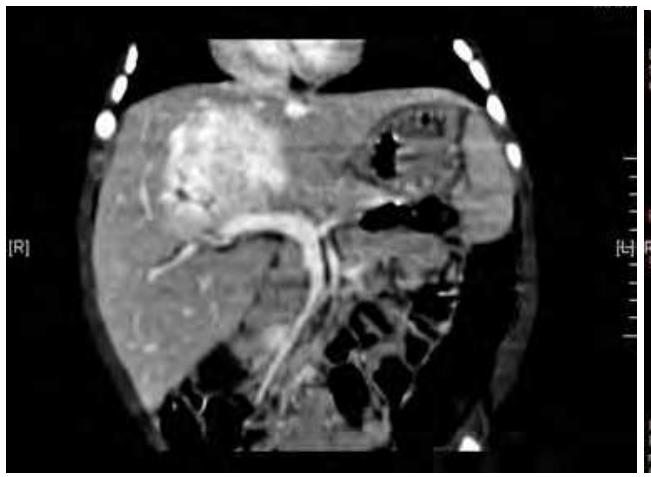

(a)

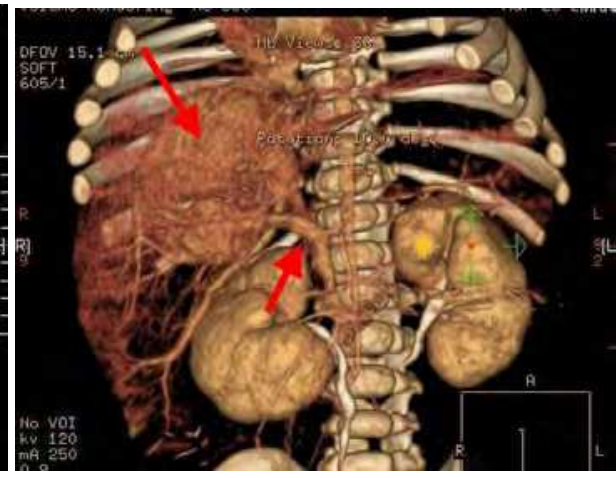

(b)

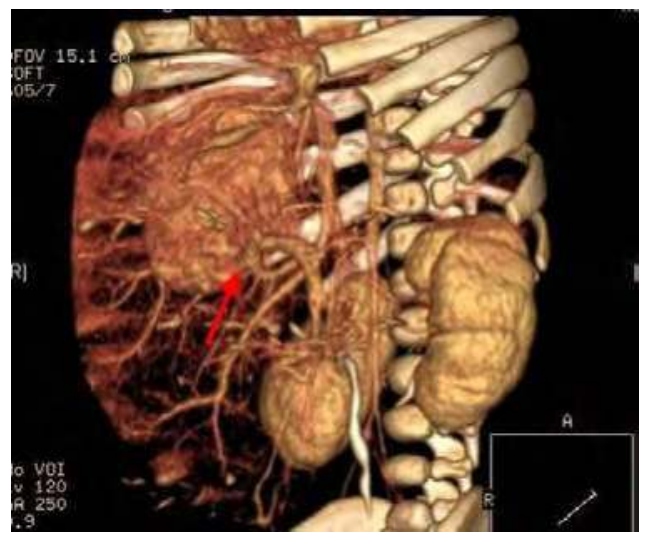

(c)

Fig. 12. a CT shows that the main portal vein is invaded by tumors at the hepatic hilum in the middle lobe. b CT 3-D imaging shows the margins of the tumors and their relationships with hepatic vessels. The arrows indicate tumors situated near the portal vein or in the middle lobe. The frontal (anteroposterior) image suggests that the main portal vein is completely embedded by the tumor. c After dynamically rotating the CT 3-D images, the branches arising from the main portal vein posterior to the tumor deviated from the tumor, indicating possible preservation of the main portal vein. This suggests that CT 3-D imaging could be used to guide the operation procedures

children with liver tumors. However, maintaining the integrity of hepatic blood vessels is a prerequisite for a successful resection, and this process is more difficult when the tumor is exceedingly large or located close to major hepatic vessels. Therefore, proper imaging of the liver is necessary and can shorten the surgical duration and increase the accuracy of the resection. Three-dimensional images can display the location of the tumor relative to blood vessels. In particular, rotatable dynamic images clearly illustrate the path and location of 
important blood vessels, thus facilitate surgical approach to the location of the tumor and determine the incision line. To some extent, three-dimensional imaging is superior to conventional enhanced CT imaging, particularly in helping the surgeon evaluate the feasibility of one-stage resection. For example, as shown in Fig.12, the tumor located at the hepatic hilum involving with the trunk of the portal vein, which may preclude a one-stage operation. Nevertheless, after dynamically rotating the three-dimensional CT images, we were able to see that a ramification originated from the trunk of the portal vein behind the tumor and leave away from the tumor; therefore, the trunk of portal vein could be reserved (Kinoshita et al..2009; Dong et al, 2007, Dong et al.,2009).

\section{Conclusions}

CT has always played a major role in the imaging of the liver. The development of multidetector row CT (MDCT) technology has helped CT to continue to excel in its already established indications (ie, hepatic lesion detection and characterization) and to add new clinical indications (ie, CT angiography, three-dimensional imaging). For children with liver tumors, proper imaging can shorten the surgical duration and increase the accuracy of the resection, especially using. three-dimensional images.

However, a major drawback of MDCT is the use of ionising radiation and, consequently, the risks of radiation-induced side effects. Therefore, reducing the radiation dose and its associated risks in children should be one of the major goals of the (paediatric) radiologist.

\section{References}

American Society of Anesthesiologists Task Force. (1996). Practice guidelines for sedation and analgesia by non-anesthesiologist: a report by the American Society of Anesthesiologists Task Force on sedation and analgesia by non-anesthesiologists. Anesthesiology,84:459-471.

Aytekin, Oto., Eric, P.\& Tamm, Janio Szklaruk.(2005) Multidetector Row CT of the Liver,Radiol Clin N Am, $43: 827$ - 848.

Bae, KT.\& Heiken, JP.(2005). Scan and contrast administration principles for MDCT, Eur Radiol,15 [suppl 5]:E46 -E59.

Bae, KT., Tao, C., Gurel, S., et al.(2007). Effect of patient weight and scanning duration on contrast enhancement during pulmonary multidetector CT angiography, Radiology,242 : 582-589.

Bae, KT., Shah, AJ., Shang, SS.et al.(2008). Aortic and hepatic contrast enhancement with abdominal 64-MDCT in pediatric patients: effect of body weight and iodine dose, AJR Am J Roentgenol, Nov;191(5):1589-94.

Bisset, GS III.\& Ball, WS.(1991). Preparation, sedation and monitoring of the pediatric patient in the magnetic resonance suite, Semin Ultrasound CT MR,2:376-378.

Caoili, EM., Cohan, RH., Korobkin, M.; et al.(2002). Urinary tract abnormalities: initial experience with multi-detector row CT urography, Radiology,222:353-360.

Chang, HJ., Jin, SY., Park, C.; et al. (2006). Mesenchymal hamartomas of the liver: comparison of clinicopathologic features between cystic and solid forms, J Korean Med Sci ,21:63-68. 
Cody, DD.(2002). AAPM/RSNA physics tutorial for residents: topics in CT. Image processing in CT, RadioGraphics,22:1255-1268.

Committee on Drugs, American Academy of Pediatrics. (1992).Guidelines for monitoring and management of pediatric patients during and after sedation for diagnostic and therapeutic procedures, Pediatrics,89:1110-1115.

Cote, CJ.(1994). Sedation for pediatric patient: a review, Pediatr Clin North Am,41:31-56.

Davey, MS.\& Cohen, MD.(1996). Imaging of gastrointestinal malignancy in childhood, Radiol Clin North Am, 34:717-742.

Dong, Q., Xu, W., Jiang, B.; et al.(2007). Clinical application of computerized tomography 3-D reconstruction imaging for diagnosis and surgery in children with large liver tumors or tumors at the hepatic hilum, Pediatr Surg Int, 23: 1045-1050.

Dong ,Q., Jiang, B., Lu, Y.; et al.(2009). Surgical management of giant liver tumor involving the hepatic hilum of children, World J Surg, Jul;33(7):1520-1525.

Donnelly, LF.\& Bisset GS III. (1998). Pediatric hepatic imaging. Radiol Clin North Am, 36:413-427.

Emre, S.\& McKenna, GJ. (2004). Liver tumors in children, Pediatr Transplant,8:632-638.

Frericks, BB., Caldarone, FC., Nashan, B.; et al.(2004).3D CT modeling of hepatic vessel architecture and volume calculation in living donated liver transplantation,Eur Radiol, 14: 326-333.

Frush, DP., Bisset, GS III.\& Hall, SC. (1996). Pediatric sedation in radiology: the practice of safe sleep, AJR,;167:1381-1387.

Frush, DP., Siegel, MJ.\& Bisset, GS 3rd.(1997). From the RSNA refresher courses: challenges of pediatric spiral CT, RadioGraphics,17 : 939-959.

Frush, DP. (2008). MDCT in children: scan techniques and contrast issues. In: Kalra MK, Sanjay S, Rubin GD (eds) Multidetector CT: from protocols to practice, 1st edn. Springer Verlag, Heidelberg, pp 331-351.

Geleijns, J., Salvadó Artells, M., Veldkamp, WJH; et al. (2006). Quantitative assessment of selective in-plane shielding of tissues in computed tomography through evaluation of absorbed dose and image quality, Eur Radiol ,16:2334-2340.

Greenberg, M.\& Filler, RM.(1997). Hepatic tumors. In: Pizzo PA, Poplack DG, eds. Principles and Practice of Pediatric Oncology, Philadelphia: Lippincott-Raven,717-732.

Helmberger, TK., Ros, PR., Mergo, PJ. et al. (1999). Pediatric liver neoplasms: a radiologicpathologic correlation, Eur Radiol ,9:1339-1347.

Jha, P., Chawla, SC., Tavri, S.et al.(2009), Pediatric liver tumors--a pictorial review, Eur Radiol, Jan;19(1):209-219.

Kinoshita, Y., Souzaki, R., Tajiri, T.,et al..(2009). A preoperative evaluation for neo-infantile liver tumors using a three-dimensional reconstruction of multidetector row $\mathrm{CT}$, Oncol Rep, Apr;21(4):881-886.

Krauss, B.\& Green, SM. (2000). Sedation and analgesia for procedures in children, N Engl J Med, 342(13):938-945

Kuhn, JP., Slovis, TL., Haller, JO.et al. (2004). Caffey's pediatric diagnostic imaging, 10th edn. Mosby,Philadelphia. 
Leswick, DA., Hunt, MM., Webster, ST; et al. (2008). Thyroid shields versus z-axis automatic tube current modulation for dose reduction at neck CT, Radiology, 249:572-580.

Maher, MM., Kalra, MK., Sahani, DV.et al,(2004).Techniques, clinical applications and limitations of 3D reconstruction in CT of the abdomen,. Korean J Radiol, JanMar;5(1):55-67.

Nievelstein, RA., van Dam, IM.\& van der Molen, AJ.(2010). Multidetector CT in children: current concepts and dose reduction strategies, Pediatr Radiol, Aug;40(8):1324-44.

Pereira, JK., Burrows, PE., Richards, HM.et al.(1993). Comparison of sedation regimens for pediatric outpatient CT, Pediatr Radiol,23:341-344.

Pobiel, RS.\& Bisset, GS, 3rd.(1995).Pictorial essay: imaging of liver tumors in the infant and child, Pediatr Radiol,25(7):495-506.

Powers, C., Ros, PR., Stoupis, C.;et al. (1994). Primary liver neoplasm: MR imaging with pathologic correlation, Radiographics, 14: 459-482

Roche, KJ., Genieser, NB.\& Ambrosino, MM. (1996).Pediatric hepatic CT: an injection rate, Radiology,26:502-507.

Roebuck, DJ.(2009). Assessment of malignant liver tumors in children,Cancer Imaging, Oct 2;9 Spec No A:S98-S103.

Sahani, D,, Saini, S., Pena, C., et al. (2002).Using multidetector CT for preoperative vascular evaluation of liver neoplasms: technique and results,AJR Am J Roentgenol Am J Roentgenol,179:53-59.

Scuza, RA.\& Narla, LD.(1992). Primary endodermal sinus tumor of the liver detected by CT, Pediatr Radiol , 22:449-450.

Shankar, VR. (2008).Sedating children for radiological procedures: an intensivist's perspective, Pediatr Radiol, May;38 Suppl 2:S213-217.

Siegel, MJ. (1998).Protocols for helical CT in pediatrics. In: Silverman PM, ed. Helical (Spiral) Computed Tomography. Philadelphia: Lippincott-Raven:179-224.

Siegel, MJ.(1999). Liver and biliary tract. In: Siegel MJ, ed. Pediatric Body CT. Philadelphia: Lippincott Williams \& Wilkins;:141-174(a).

Siegel, MJ.(1999). Practical CT techniques In: Siegel MJ, ed. Pediatric Body CT. Philadelphia: Lippincott Williams \& Wilkins:1-41(b).

Siegel, MJ.(2001). Pediatric liver imaging,Semin Liver Dis,May;21(2):251-269.

Siegel, MJ., Chung, EM.\& Conran, RM.(2008). Pediatric liver: focal masses,Magn Reson Imaging Clin N Am, Aug;16(3):437-452.

Siegel ,MJ. (2008).Practical CT techniques. In: Siegel MJ (ed) Pediatric body CT, 2nd edn. Lippincott Williams \& Wilkins, Philadelphia, pp 1-32.

Strain, JD., Campbell, JB., Harvey, LA.et al.(1988). IV Nembutal: safe sedation for children undergoing CT, AJR, 151:955-959.

Stringer, MD.\& Alizai, NK. (2005). Mesenchymal hamartoma of the liver: a systematic review, J Pediatr Surg ,40:1681-1690.

Taghon, TA., Bryan, YF.\& Kurth, CD. (2006). Pediatric radiology sedation and anesthesia, Int Anesthesiol Clin, 44:64-79. 
Vollmar, SV.\& Kalender, WA. (2008).Reduction of dose to the female breast in thoracic CT: a comparison of standard-protocol, bismuthshielded, partial and tube-currentmodulated CT examinations, Eur Radiol,18:1674-1682 


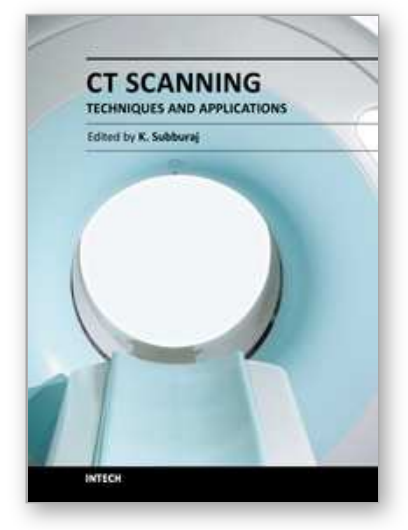

\author{
CT Scanning - Techniques and Applications \\ Edited by Dr. Karupppasamy Subburaj
}

ISBN 978-953-307-943-1

Hard cover, 348 pages

Publisher InTech

Published online 30, September, 2011

Published in print edition September, 2011

Since its introduction in 1972, X-ray computed tomography (CT) has evolved into an essential diagnostic imaging tool for a continually increasing variety of clinical applications. The goal of this book was not simply to summarize currently available CT imaging techniques but also to provide clinical perspectives, advances in hybrid technologies, new applications other than medicine and an outlook on future developments. Major experts in this growing field contributed to this book, which is geared to radiologists, orthopedic surgeons, engineers, and clinical and basic researchers. We believe that CT scanning is an effective and essential tools in treatment planning, basic understanding of physiology, and and tackling the ever-increasing challenge of diagnosis in our society.

\title{
How to reference
}

In order to correctly reference this scholarly work, feel free to copy and paste the following:

Qian Dong and Jingjing Chen (2011). CT Scan of Pediatric Liver Tumors, CT Scanning - Techniques and Applications, Dr. Karupppasamy Subburaj (Ed.), ISBN: 978-953-307-943-1, InTech, Available from: http://www.intechopen.com/books/ct-scanning-techniques-and-applications/ct-scan-of-pediatric-liver-tumors

\section{INTECH}

open science | open minds

\section{InTech Europe}

University Campus STeP Ri Slavka Krautzeka 83/A 51000 Rijeka, Croatia Phone: +385 (51) 770447

Fax: +385 (51) 686166 www.intechopen.com

\section{InTech China}

Unit 405, Office Block, Hotel Equatorial Shanghai No.65, Yan An Road (West), Shanghai, 200040, China 中国上海市延安西路65号上海国际贵都大饭店办公楼 405 单元 Phone: $+86-21-62489820$

Fax: $+86-21-62489821$ 
(C) 2011 The Author(s). Licensee IntechOpen. This chapter is distributed under the terms of the Creative Commons Attribution-NonCommercialShareAlike-3.0 License, which permits use, distribution and reproduction for non-commercial purposes, provided the original is properly cited and derivative works building on this content are distributed under the same license. 